\title{
Interacción multinivel en estudiantes de Ingeniería Química de la Universidad de Costa Rica
}

\author{
Multilevel Interaction in Chemical Engineering Students from University of Costa Rica

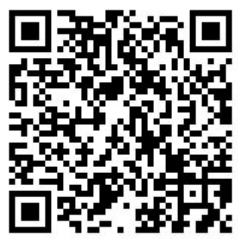 \\ Jenny Andrea Calderón-Castro ${ }^{7}$
Universidad de Costa Rica
San José, Costa Rica
jenny.calderoncastro@ucr.ac.cr \\ Leonardo Garro-Mena² \\ Universidad de Costa Rica \\ San José, Costa Rica \\ leonardo.garromena@ucr.ac.cr
}

Recibido 31 de octubre de 2014 • Corregido 11 de octubre de 2015 • Aceptado 3 de noviembre de 2015

Resumen. La presente investigación estudia el efecto que la interacción multinivel, en estudiantes de diferentes niveles de la carrera de Ingeniería Química de la Universidad de Costa Rica, tiene sobre el aprendizaje y el desarrollo de habilidades. Los grupos entre los cuales se estableció contacto fueron Análisis gráfico, de segundo año de carrera, y Control e instrumentación, de quinto año de carrera. La metodología utilizada es cualitativa basada en el estudio de caso, se utilizaron instrumentos tales como la observación y recolección de datos, la bitácora del estudiantado, entrevistas y cuestionarios dirigidos a la población participante. Se observó que la interacción genera una mejor comprensión de las temáticas de los cursos, además de promover el desarrollo de habilidades sociales. Dados los resultados positivos de la experiencia, se recomienda generar espacios para la interacción no solo entre estudiantes, sino entre personal docente e incluso a nivel industrial.

Palabras claves. Interacción multinivel, aprendizaje en equipos, estrategia didáctica, transversalidad curricular.

Abstract. This research studies the effect of multilevel interaction among students of different levels from the chemical engineering career at the University of Costa Rica regarding the development and learning of skills. The groups among whom we contacted were second year graphic analysis students and fifth year control and instrumentation students. The methodology used is qualitative case study-based; instruments used were observation and data collection, students' logbook, interviews and questionnaires geared towards the participating population. It was observed that interaction generates a better understanding of the courses' subject matter, as well as promote the development of social skills. Given the positive results of the experience, it is recommended to create spaces for interaction not only between students, but between teachers and even at industrial level.

Keywords. Multilevel interaction, team learning, teaching strategy, multidimensional curriculum.

\footnotetext{
${ }^{1}$ Licenciada en Ingeniería Química y egresada de la Maestría Profesional en Ingeniería Química con énfasis en Ingeniería Ambiental de la Universidad de Costa Rica. Es docente e investigadora en la Universidad de Costa Rica, participa en proyectos de Acción Social.

${ }^{2}$ Licenciado en Ingeniería Química y Magíster en Ingeniería Química con énfasis en Ingeniería Ambiental. Es investigador y docente en la Universidad de Costa Rica, miembro de la red para la innovación docente RedIC-UCR, de la Universidad de Costa Rica, ha realizado publicaciones en el ámbito de la docencia y la difusión científica, además trabaja como consultor externo en control de procesos e ingeniería ambiental.
} 
doi: http://dx.doi.org/10.15359/ree.20-1.19

URL: http://www.una.ac.cr/educare

CORREO: educare@una.cr

Las carreras universitarias, por lo general, basan su desarrollo en un plan de estudios en el cual se estructuran, en cierta secuencia, una determinada cantidad de cursos. Posner (1974) realizó un estudio en el que resume la estructura de los planes de estudios recomendada por los teóricos de la época. En sus conclusiones determina un esquema compuesto por la temporalidad, que definen si las asignaturas son horizontales o verticales, y por la "comunalidad", que determina si los contenidos son repetitivos, relacionados o no relacionados. Es recurrente, en esta forma de estructura, el problema de lo que podríamos llamar islas de contenido, que ocurren cuando en un curso se desarrollan temas o habilidades, los cuales, dependiendo del conocimiento previo y de la familiaridad que el estudiantado tenga, puede que no logre ligar con una aplicación inmediata, por lo cual, dichos temas o habilidades se tiñen de cierto nivel de irrelevancia. Esta problemática es abordada por Efland (2002), quien afirma que si la educación tiene por objetivo desarrollar al máximo el potencial cognitivo de quienes desempeñan el rol de aprendientes, entonces se deben encontrar formas para integrar los conocimientos de las diferentes materias $y$, de esta manera, lograr un entendimiento más completo del que se lograría con los contenidos aislados. Illingworth (2010) Ilama a este aislamiento temático "splendid isolation" (el gran aislamiento) en referencia a un periodo de la historia británica; con esto da a entender que tal aislamiento podría ser insostenible y que quizá deberíamos crear conexiones valiosas entre diferentes materias a lo largo del currículo.

Uno de los cursos que sufren las consecuencias de esta forma de organizar las carreras universitarias es el curso Análisis Gráfico, el cual se encuentra ubicado en el primer ciclo del segundo año de la carrera de Ingeniería Química y es el primer curso de la carrera, antes de él, el estudiantado solamente habrá llevado los cursos de formación básica en ciencias impartidos por otras escuelas como cursos de servicio.

El curso de Análisis Gráfico se caracteriza por ser un curso de Dibujo Técnico, donde se desarrollan las habilidades de dibujo por medio de programas de dibujo y diseño asistido por computadora o CADD (por sus siglas en inglés) y en donde se sientan las bases para el dibujo de los planos de Ingeniería Química; sin embargo, según lo define el Reglamento al Título I de la Ley Orgánica del Colegio de Ingenieros Químicos y Profesionales Afines de Costa Rica (reglamento que rige el futuro quehacer profesional del estudiantado) un plano se define como una "Representación gráfica a determinada escala de una planta, proceso, máquina o equipo..." por lo tanto, los planos ameritan conocer conceptos de diseño, implementación y construcción de equipos y procesos, los cuales serán adquiridos y discutidos en cursos posteriores.

Así pues, al tratarse del primer curso de la carrera se hace evidente la importancia de brindar una introducción a esta, ya que el estudiantado no entiende, al nivel de su segundo año, el lenguaje y los conceptos usuales utilizados en la Ingeniería Química, debido a que no han recibido un curso introductorio a la especialidad. Pero, a pesar del esfuerzo, resulta difícil tratar de explicar, aunque sea de forma general, los principios y elementos necesarios en un curso sin los conocimientos teóricos previos. 
Por esto, el curso de Análisis tiene un reto significativo, ya que el estudiantado debe aprender a dibujar planos de proyectos que no entienden, por lo que tiende a convertirse en una copia de intrincadas configuraciones de líneas que no tienen significado sino hasta cursar la carrera completa.

Por otra parte, el curso Control elnstrumentación de Procesos seencuentra en el primer ciclo del quinto año de carrera, finalizando casi el currículo de la Licenciatura. En el curso de Control e Instrumentación de Procesos el estudiantado debe desarrollar las capacidades de comprensión de la dinámica de los procesos químicos, así como la capacidad de diseñar sistemas de control de estos mismos y la instrumentación adecuada. En este curso, el efecto de la irrelevancia intrínseca es menor, ya que el grupo de estudiantes cuenta con una gran comprensión de los procesos químicos, por lo que le es más fácil visualizar los sistemas de control que se diseñan y su respectiva aplicación. A pesar de ello, la implementación de sistemas de control a nivel nacional suele enfrentarse, en la mayoría de los casos, con un grave inconveniente: el factor humano. Y es que en la formación profesional en Ingeniería Química no se potencia la formación de las capacidades para lidiar con el factor humano, con la psicología de personal operador de las plantas o con los problemas sociales que se generan en el ambiente laboral. Por ello muchos proyectos, a pesar de tener un sólido diseño técnico, terminan fracasando, pues no se toma en cuenta la cultura de la empresa, la micro sociedad que es un grupo de personal trabajador.

En consecuencia, el curso de Control e Instrumentación de Procesos adolece de una carencia de formación en lo que respecta a la adecuada implementación de sistemas de control más allá de lo técnico, como el manejo de personal, la resolución de conflictos, la interacción con personas que tienen conocimientos en muy diferentes niveles a los propios (técnicos, operarios, aprendices, etc.). Además, la utilización de paquetes CADD en la formación del estudiantado es bastante nueva, por lo que en niveles, como quinto año a la fecha, el estudiantado recibió menos horas de trabajo de dibujo digital que el grupo actual, lo que amerita que para preparar planos de sus diseños sea necesario aprender habilidades digitales que no se impartían cuando llevaron el curso en segundo año.

\section{Problemática}

Dado el contexto en el que se encuentran los cursos de Control e Instrumentación y Análisis Gráfico y las limitaciones cognitivas que se generan en el aislamiento de materias propias del currículo actual (Efland, 2002), en esta investigación se plantea una interacción multinivel con los grupos de estudiantes de los cursos mencionados. Esto, con el objetivo de determinar: ¿Cómo influye la interacción multinivel, entre estudiantes de cursos de diferentes niveles de la carrera de Ingeniería Química, en el aprendizaje y las habilidades desarrolladas por los grupos de estudiantes? 
doi: http://dx.doi.org/10.15359/ree.20-1.19

URL: http://www.una.ac.cr/educare

CORREO: educare@una.cr

\section{Interacción multinivel en sistemas de educación formal}

Diversas investigaciones han abordado temas afines a la interacción multinivel en los sistemas de educación formal, en este ámbito se encuentran publicaciones como la de Pardini (2005), que analiza el declive en la popularidad de los sistemas educativos multiedad en los Estados Unidos. La razón de tal decadencia, según Pardini (2005), está en discusión, sin embargo, pareciera deberse a cuestiones políticas y administrativas (se quiere favorecer el programa No Child Left Behind, el enfoque de multiedad es muy afectivo y no es bien aceptado o el hecho de que se requiera más trabajo por parte del cuerpo docente son algunas de las razones propuestas) más que a los resultados obtenidos en la implementación. Sin embargo, cuando lo evaluado son los resultados obtenidos, el enfoque multinivel se mantiene, incluso se potencia. Esto último quizá debido a que la fragmentación por edad en los centros de enseñanza fue un desarrollo que se llevó a cabo por conveniencia administrativa, de esta forma el material didáctico se podía estandarizar y el cuerpo docente no tenía que dominar tantos contenidos al mismo tiempo, esta aproximación es antinatural y, a pesar de facilitar la administración de los sistemas educativos, lo hizo en detrimento de su eficacia (Pardini, 2005).

Por otra parte, en lo que respecta a la educación superior, se han desarrollado experiencias que buscan trascender las materias, por ejemplo, Pop-lliev y Nokleby (2011) llevaron a cabo una experiencia en la cual se integran los conocimientos de dos cursos diferentes para desarrollar un proyecto, el objetivo es afrontar el diseño en una carrera de ingeniería por medio de la integración de conceptos básicos a la resolución de un problema de mayor complejidad. Esta iniciativa busca la integración de conocimientos, pero lo hace sin modificar la estructura del ambiente formativo, cada equipo de estudiantes trabaja en solitario, lo que se integra son los conocimientos, no las personas. Una experiencia similar realizaron Hickman y Kiss (2010) en el campo de la enseñanza del arte, en este caso se presenta una estrategia que trasciende al aula y los contenidos, pues se expone a los equipos de estudiantes a temáticas diversas, aunque tampoco integra personas con diversos conocimientos, solo contenidos.

En las experiencias recién expuestas, las fronteras de las materias o de las temáticas se traspasan por medio de la construcción de una estrategia en la cual el estudiantado debe integrar conocimientos de diversas materias, e incluso disciplinas. Un caso diferente es presentado por Kapadia (2010), quien expone una estrategia docente para manejar el concepto de riesgo. En este caso dos profesores de disciplinas diferentes interactúan para plantear actividades en las que se refleje esa integración disciplinar, de tal forma que las actividades de aprendizaje incrementen su significancia. Kapadia (2010) propone una interacción entre personas con conocimientos diversos, pero lo hace al nivel del equipo docente, no del estudiantado. 
En el caso de Gardner, Tuchman y Hawkins (2010) la integración es más compleja, pues diseñan una estrategia sustentada en aprendizaje basado en problemas para tres cursos de la carrera de trabajo social, de tal forma que el problema planteado es resuelto por equipos de trabajo compuestos por estudiantes de los tres cursos. La justificación planteada por los autores es que la pobreza en comunidades rurales, que es el tema en el que se basa el problema, es compleja y, por lo tanto, requiere de una aproximación integral. Cada equipo de trabajo tiene objetivos claros por cumplir, tanto a nivel de equipo local como en la globalidad, donde se integran los tres cursos.

Todas estas iniciativas, más allá de sus diferencias en estructura y sus particularidades, tienen un elemento en común, un elemento que anteriormente señaló Cross (1999), quien propone que el aprendizaje se basa en crear conexiones "learning is about making conections [El aprendizaje se trata de hacer conecciones]". Dichas conexiones se establecen en diferentes niveles, ya sea neuronal, en donde el aprendizaje implica la creación de sinapsis; ya sea mental, como la conexión entre dos conceptos aparentemente aislados o incluso un nivel donde se mezclan experiencias y conceptos, como cuando se logra vislumbrar la conexión entre una abstracción y una aplicación práctica (Cross, 1999).

De esta manera, si se quiere realizar una interacción multinivel para resolver problemas complejos, se debe tomar en cuenta algunas características para el diseño de la estrategia. Lauer (2000) especifica que para llamar realmente multinivel a una clase, no solo se requiere que esté compuesta por estudiantes de diferentes edades y diferentes niveles, sino que haya una conciencia por parte del cuerpo docente de tal forma que el estudiantado se pueda beneficiar de tal interacción. En resumen, las clases multinivel no pueden ser accidentes, sino estrategias didácticas planeadas.

Además, la estructura y planeación de las actividades multinivel buscan lograr aprovechar las ventajas de la interacción; a este respecto Anderson (1987) plantea un escenario en el que las barreras que separan a docentes y estudiantes deben ser superadas, en donde cada estudiante debe ponerse en contacto con estudiantes de otros niveles, fomentar el contacto con los de su mismo nivel, interactuar con estudiantes que muestren otros intereses, con individuos adultos, en fin, que cada persona, en su proceso de formación, se exponga a una diversidad de situaciones amplia y rica (Anderson, 1987). Carter (2005), por ejemplo, discute sobre una comunidad de aprendizaje, estudiantes de primaria de los tres primeros niveles en un mismo salón, de tal forma que el proceso educativo emule una familia, en la que se coopera y crece en conjunto; todo esto por medio del uso de situaciones cotidianas para el estímulo. Pues una situación cotidiana en un aula de clases es toda una novedad. 
doi: http://dx.doi.org/10.15359/ree.20-1.19

URL: http://www.una.ac.cr/educare

CORREO: educare@una.cr

\section{Metodología}

El diseño de la investigación se basó en la metodología cualitativa a través de la utilización del método de estudio de caso. El interés primordial de este estudio radicó en colocar a dos grupos de diferentes niveles, del plan de estudios de la carrera de Ingeniería Química de la Universidad de Costa Rica, a realizar en conjunto su trabajo final de curso, con el fin de tener una experiencia multinivel y analizar el proceso de aprendizaje a partir de esa interacción.

En el diseño de la investigación se plantearon varias etapas, durante la primera, la fase exploratoria, se realizaron entrevistas no estructuradas al estudiantado, tipo conversatorio donde las preguntas se fueron generando según los datos que se iban recolectando, y se planteó la problemática del estudio. En la segunda etapa, se realizaron tomas de datos por medio de cuestionarios y guías de observación, además del análisis preliminar de esos datos. Por último, en una tercera fase, se entrevistó a dos estudiantes después de dos años de realizada la investigación de campo, con el fin de medir su impacto. El proceso investigativo se realizó durante los años 2012 a 2014.

Se conformó un equipo docente compuesto por el profesor Leonardo Garro del curso IQ-0517 Control e Instrumentación de Procesos y la profesora Jenny Calderón del curso IQ0200 Análisis Gráfico para Ingeniería Química. La primera tarea se basó en escribir la guía de trabajo, las instrucciones y la manera de conformar los equipos de trabajo de los dos cursos. Luego se procedió a escoger un problema abierto de ingeniería, donde la respuesta conlleva a múltiples soluciones, para la resolución conjunta y dárselo al estudiantado. La conformación de los equipos dentro de los cursos se realizó de manera abierta, de tal forma que el estudiantado se agrupó por medio de afinidades.

El trabajo final o proyecto final del curso se inició al principio de cada semestre en el que se realizó la experiencia, por lo que, el estudiantado tuvo la oportunidad de relacionarse por casi cuatro meses en total. Durante ese tiempo, existieron reuniones semanales llamadas Avances, donde el equipo presentaba el progreso del proyecto, sus dudas y dificultades.

Es importante resaltar que las técnicas e instrumentos utilizados para la recolección de datos fueron: primero, la observación, donde el equipo docente realizaba interpretaciones propias de lo observado de manera individual; segundo, en la fase de avances, las observaciones se realizaron de manera conjunta; tercero, el estudiantado llevaba consigo una bitácora del proceso, donde, a manera de diario, recababan la información del proceso de aprendizaje, sus dudas y sus sugerencias con el fin de ser revisadas cada semana por el equipo docente. Y cuarto, la utilización de un cuestionario al final del trabajo y entrevistas no estructuradas.

Con respecto al proceso en el proyecto final del curso y su gestión por equipos, existieron varios momentos en los que el estudiantado tuvo que resolver en conjunto algunas situaciones, esos momentos se explican a continuación:

6

Jenny Andrea Calderón-Castro y Leonardo Garro-Mena

(c) (1) (3)

Artículo protegido por licencia Creative Commons 


\section{a) Elección del tema del proyecto final}

El tema a desarrollar por el estudiantado se plantea de tal forma que cuente con una serie de características que lo doten de relevancia, interés, actualidad y significancia. Para que el proyecto sea relevante se elige un tema de trascendencia, tal que se enmarque en la aplicación de la Ingeniería Química, que le haga sentir identificado con sus carreras y que le permita vislumbrar aplicaciones prácticas de sus conocimientos, por ejemplo, el diseño de una biorrefinería o lograr suplir de combustibles de fuentes renovables o biocombustibles a una empresa de autobuses. Para despertar el interés del estudiantado, el proyecto se plantea de tal forma que sea un reto, pero que cada equipo de estudiantes sienta que puede lograrlo, aunque parece ser difícil. La actualidad se logra eligiendo problemáticas que traten situaciones en boga o con soluciones novedosas en al ámbito de desarrollo del estudiantado, cabe destacar que los temas resueltos por los equipos se centraban en la problemática ambiental y la cercanía del 2021 y la meta de carbono neutralidad adquirida por Costa Rica. Por último, la significancia es tratada enmarcando al proyecto en el ámbito nacional, en las tendencias mundiales, en problemas abiertos de la ingeniería o en algún marco similar que dote a la resolución de un significado más allá del simple ejercicio didáctico.

\section{b) Documento de especificaciones}

Luego de que se ha elegido el tema con las características citadas anteriormente, se procede a confeccionar el documento con las especificaciones que deberán respetarse para desarrollar el proyecto. Dicho documento se realiza de tal forma que establezca una base sobre la cual se estandaricen los trabajos, además de ser una guía que oriente a los equipos hacia la resolución en tanto que se cumplen los objetivos del trabajo. Por otra parte, el documento es lo suficientemente abierto para que no se limite la investigación, creación, ingenio e imaginación de cada equipo de estudiantes.

\section{c) Bitácora}

Luego de que se ha elegido el problema y se han conformado y emparejado los equipos de trabajo de ambos cursos, se abre una bitácora con la cual se persiguen tres fines básicos. Primero, la bitácora pretende emular el desarrollo de un proyecto de ingeniería real, en el cual el control se hace, precisamente, por este medio. Segundo, la bitácora funge como evidencia del proceso de construcción de la solución del problema, en ella se documentan las dudas, descubrimientos, ideas y todo aquello que sea de relevancia. Finalmente, como tercer punto, la bitácora persigue obtener transparencia en la colaboración, cada reunión que los equipos lleven a cabo debe quedar documentada, así como los compromisos y acuerdos. Además, se trata de un instrumento de toma de datos que cada equipo confecciona para revisión por el equipo docente. 
doi: http://dx.doi.org/10.15359/ree.20-1.19

URL: http://www.una.ac.cr/educare

CORREO: educare@una.cr

\section{d) Avances de trabajo}

A lo largo del trabajo los equipos presentan avances de lo realizado hasta el momento, la dinámica de la presentación del avance es tal que cada equipo expone lo que ha hecho el otro, esto es, el equipo de Análisis Gráfico presenta los avances del equipo de Control e Instrumentación y viceversa, con una fuerte interacción entre equipos. Cabe destacar que los avances de trabajo se realizan por parte del equipo al equipo docente. A continuación se presentan los avances conjuntos, las limitaciones encontradas, las soluciones propuestas y la sensación general de cómo el trabajo avanza. Así como la resolución de dudas por parte del profesorado y la intervención de este para encaminar el proyecto si se está alejando del objetivo.

\section{e) Presentación final}

El trabajo finaliza con una exposición simultánea tipo feria en la que cada equipo expone la parte de la resolución del problema en la que trabajó. De esta forma, los equipos de Control e Instrumentación exponen la simulación, los modelos del proceso y el comportamiento dinámico. Por su parte, los equipos de Análisis Gráfico exponen los planos, el proceso en general (contemplado en el diagrama de flujo de proceso) y el comportamiento del sistema de control, que se encuentra en el diagrama de equipo tubería e instrumentación; la información técnica requerida para dicha exposición habrá sido adquirida en la interacción del diseño de su contraparte de Control e Instrumentación. La exposición es abierta y se invita a estudiantes y docentes de la Escuela, de tal forma que puedan interactuar y compartir las impresiones de la experiencia.

\section{Estructura e interacción}

En la figura 1 se presenta, a modo de mapa conceptual, la estructura e interacción de los principales temas abarcados en los cursos involucrados en el presente estudio. Como se dijo anteriormente, uno de los objetivos del curso Análisis Gráfico es servir de curso introductorio a la Ingeniería Química; sin embargo, el curso posee la problemática de estar en el segundo año de carrera y el estudiantado no posee los conocimientos básicos para desarrollar una comprensión profunda de algunas de las actividades que se llevan a cabo, como lo son la comprensión de procesos y confección de planos. Al integrar ambos cursos, se logra articular, por medio de la interacción, una mejor comprensión por parte de las personas matriculadas en Análisis Gráfico, ya que están en constante exposición con sus homólogos y homólogas de Control e Instrumentación, quienes les brindan explicaciones constantes y asesoría para que logren comprehender los procesos, de tal forma que la confección de los planos se realice sin mayores problemas. La asimilación, de parte del estudiantado de Control e Instrumentación de las herramientas de dibujo digital, se logra por medio de la revisión constante del material que va produciendo la contraparte de Análisis Gráfico, ya que se le entrega la total responsabilidad de la evaluación técnica a la población estudiantil con mayor experiencia, obteniéndose así un verdadero intercambio de conocimientos. 
doi: http://dx.doi.org/10.15359/ree.20-1.19

URL: http://www.una.ac.cr/educare

Nótese la interacción entre los cursos que se explicita en la Figura 1, los diagramas de flujo de proceso (DFP) representan una serie de elementos que, en su conjunto, abstraen los aspectos operativos y funcionales de un proceso; en Control e Instrumentación dichos diagramas son esenciales, pues a partir de ellos se inicia con el diseño de los sistemas de control. Para Análisis Gráfico, los DFP adquieren significado cuando, en la interacción con los equipos de Control e Instrumentación, experimentan la construcción de una solución al problema planteado en las especificaciones; por sí solos, los equipos de Análisis Gráfico no cuentan con los conocimientos para resolver el problema planteado, quizá ni siquiera para comprender la solución encontrada, esto se logra por la interacción sinérgica que se forma transgrupalmente.

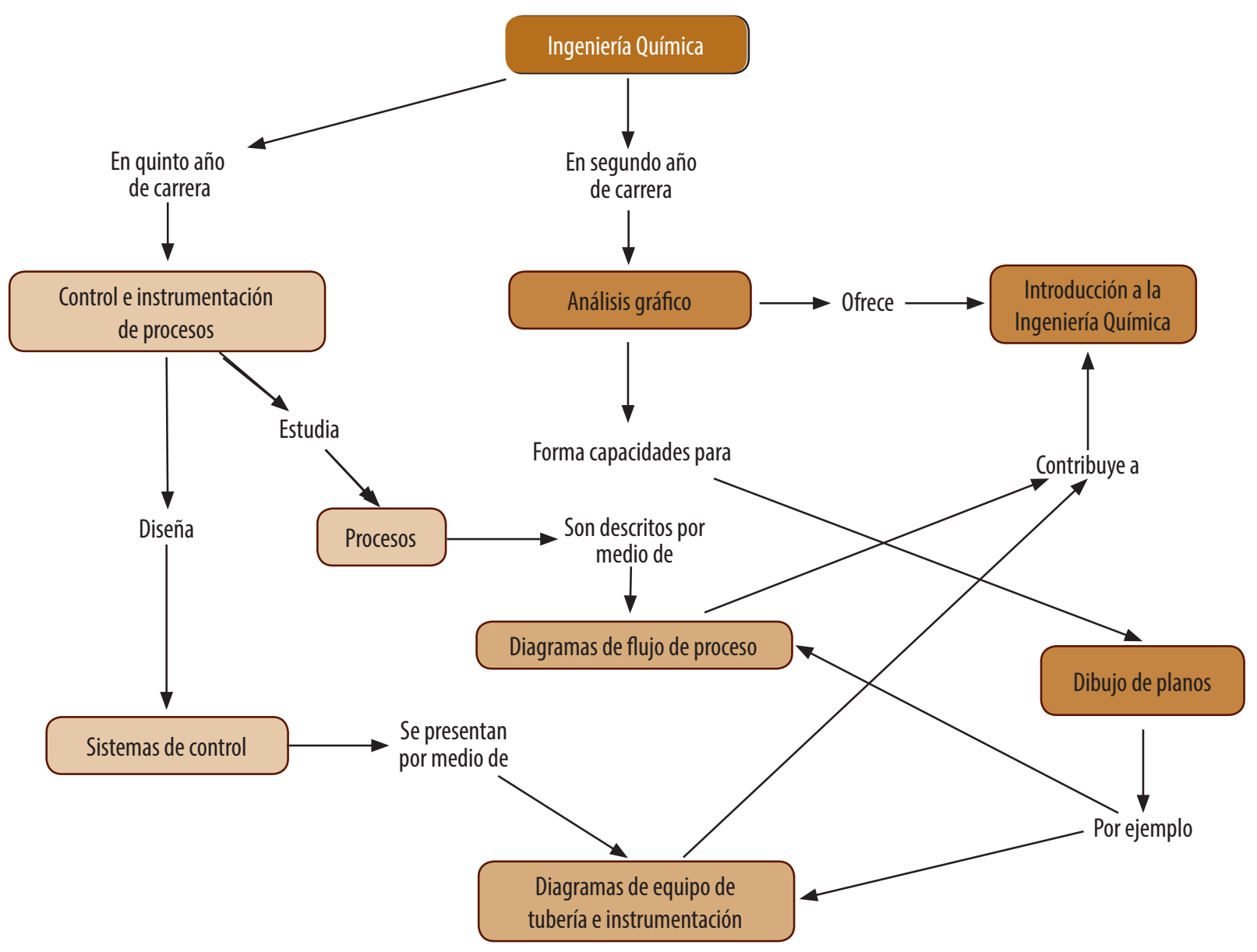

Figura 1. Estructura de la interacción entre cursos. Elaboración propia. 
doi: http://dx.doi.org/10.15359/ree.20-1.19

URL: http://www.una.ac.cr/educare

CORREO: educare@una.cr

Porsu parte, los diagramas de equipo tubería einstrumentación (DETI) son la representación de un sistema de control, ambos planos en conjunto con el proceso de resolución, la interacción transgrupal, las asignaciones y avances de trabajo logran desarrollar en cada estudiante de los equipos de Análisis Gráfico una concepción de lo que es la Ingeniería Química, de la utilidad de los planos que dibujan. Se logra, con ello, un curso, en el caso de Análisis Gráfico, que abre las puertas de la carrera a quienes recién ingresan a ella, por medio de una Introducción a la Ingeniería Química, además, por supuesto, de brindarles herramientas que utilizarán durante el resto de su formación. Y, por otra parte, un curso, en el caso de Control e Instrumentación, que abre las puertas a la población estudiantil a lo que será su ambiente laboral, en el que necesitará más herramientas que las estrictamente técnicas o de conocimiento.

\section{Resultados y análisis}

En este apartado seexponen los principales resultados obtenidos a partirdelas observaciones de la población estudiantil en estudio y de los resultados de los cuestionarios y entrevistas.

Como se mencionó, existe una problemática alrededor del aprendizaje aislado en grupos de un mismo nivel de estudios, no solo en primaria y secundaria, sino también existe la preocupación a nivel universitario. Debido a esto el equipo docente preparó una estrategia didáctica en la cual la población en estudio, estudiantes de segundo y quinto año de la carrera de Ingeniería Química de la Universidad de Costa Rica, pudieran interrelacionarse con un objetivo en común: hacer su trabajo final de curso. En una primera etapa del desarrollo del proyecto, el equipo docente realizó observaciones, en las que su función fue la de dirigir el transcurso del proyecto y resolver dudas técnicas, mientras realizaban toma de datos cualitativos. Algunas de esas observaciones se detallan a continuación.

En primera instancia se observó que el estudiantado mantuvo siempre expectativas altas con respecto al interés en el trabajo, esto fue novedoso, ya que por experiencias anteriores el trabajo es laborioso y con el tiempo decae el interés. Cuando se realizan conversaciones durante los avances del proyecto, se encuentra que la mayoría del estudiantado de segundo año se encuentra motivado, pues el proyecto se comprende en gran medida, aún siendo de un nivel de último año de carrera, además de que se acompaña por estudiantes de niveles superiores que se esfuerzan por explicarles los conceptos que no entienden. En segunda instancia, existió una gran motivación por realizar amistad con personas que estaban a punto de graduarse y se encontró que era muy común que se mantuvieran conversaciones respecto al desarrollo de la carrera, cómo eran los cursos siguientes y lo que podían esperar de ellos. Se observó que el trabajo final generó un espacio en donde el estudiantado tuvo la oportunidad de interactuar más allá de la formalidad exigida por el trabajo final, creando vínculos afectivos con otras personas. Pardini (2005) comenta que este tipo de relaciones propician el desarrollo de habilidades sociales y de liderazgo en el estudiantado, las cuales fueron observadas en el transcurso del proyecto en la población en estudio. 
Por otro lado, se visualizó la problemática del trabajo en equipos y la necesidad de comunicarse efectivamente, además de la solución de los problemas, no solo de ingeniería sino también de índole personal. El uso del tiempo y la planificación, pues al estar en niveles diferentes, se encuentran llevando cursos diferentes, y en horarios distintos, por lo que el estudiantado debió resolver el problema de la comunicación entre las personas y el equipo mismo. Así, se observó cómo el lenguaje técnico utilizado por el estudiantado de segundo nivel, fue adoptando términos y se fueron apropiando de conceptos, los cuales, de no ser por la interacción, no conocerían hasta a un nivel superior. Asimismo, los grupos de segundo lograron darle contenido al dibujo de un plano que normalmente no se entiende por la falta de conocimientos.

Desde la perspectiva de los grupos de quinto, se observó cómo entraron en conciencia de cuánto habían avanzado en la carrera, al recordar y simpatizar con los de segundo. Además, necesitaron crear un lenguaje sencillo para explicar conceptos cuando los de segundo los interrogaban. También, aprendieron el manejo del personal (como será en una futura vida profesional), tuvieron que enfrentar a personas que no tenían el mismo conocimiento que ellos, y explicarles y pedir tareas y laborales sobre el proyecto que realizaban por medio del lenguaje sencillo desarrollado.

En el transcurso de la investigación, la bitácora del proyecto se volvió un instrumento de recolección de datos de primera mano, ya que los equipos de trabajo la utilizaron a modo de diario, escribiendo cada actividad que realizaban, fechas de reuniones, los lugares y medios favoritos y sus dudas y sugerencias en cuanto al proyecto. Para el equipo docente se volvió de vital importancia para observar cómo se comunicaban los grupos de estudiantes, las dudas que se iban generando en el proceso y cómo se iban resolviendo con las personas de nivel superior.

Al mismo tiempo, se identifica el uso de medios de comunicación como grupos en Facebook, correos electrónicos, mensajes de texto, reuniones grupales presenciales y no presenciales en chat de video. Muchos grupos aportaron sus observaciones, pues les placía llenarla para mostrar sus avances al equipo docente. Por otra parte, se pudo evaluar la interacción de cada miembro, e intervenir con eficacia si alguna persona fallaba en sus labores. Además, fue el instrumento para medir la velocidad de adquisición de conocimientos, notándose que en cursos anteriores el estudiantado llegaba con más dudas, pues no tenían a quien preguntar de no ser a su docente de curso. Por lo que se percibió que primero fue lento y torpe, hasta volverse ágil y mucho más complejo. Por último, se observó que el compromiso con el proyecto aumentó, pues las acciones de una persona repercutían en el equipo, por ejemplo, tenían que ponerse de acuerdo para que todos firmaran la bitácora.

Al finalizar el trabajo se realizó una recolección de información por medio un cuestionario dirigido a la población de estudio, dicha recolección se hizo por medio de un formulario electrónico a un total de 32 estudiantes, de los cuales 14 pertenecían al curso Análisis Gráfico y 18 al curso Control e Instrumentación. El formulario buscaba, primero, obtener una evaluación 
doi: http://dx.doi.org/10.15359/ree.20-1.19

URL: http://www.una.ac.cr/educare

CORREO: educare@una.cr

de la experiencia por parte de quienes se desempeñaron como aprendientes en esta misma. Segundo, extraer las características de mayor impacto que la actividad generó en el grupo de estudiantes. $Y$ tercero, generar un compendio de sugerencias para mejorar la estructura y aplicación en futuros grupos de estudiantes. A continuación se detallan los resultados.

\section{Evaluación estudiantil}

La evaluación que cada estudiante realizó sobre la actividad se dividió en dos frentes: el primero, la percepción sobre las habilidades desarrolladas y su relevancia para la futura vida profesional; el segundo, la pertinencia y tino de la estructura del trabajo, y su extrapolación a otras áreas de la carrera. Con respecto a la percepción sobre las habilidades desarrolladas y su relevancia para la futura vida profesional, se ejecutaron tres preguntas, las cuales se presentan en las figuras 2, 3 y 4 . En primera instancia se consulta sobre si se considera que el trabajo en conjunto ha contribuido a la formación profesional del estudiantado, los resultados se muestran en la figura 2. El $47 \%$ del estudiantado considera que la actividad contribuyó mucho a su formación profesional, en tanto que un $41 \%$ consideró que hubo algo de contribución. Es importante notar que el porcentaje de estudiantes que consideran que el trabajo conjunto no contribuyó con su formación profesional es de $0 \%$, por lo que, el $100 \%$ considera que hubo contribución en alguna medida.

Análisis gráfico

Control e instrumentación

Total

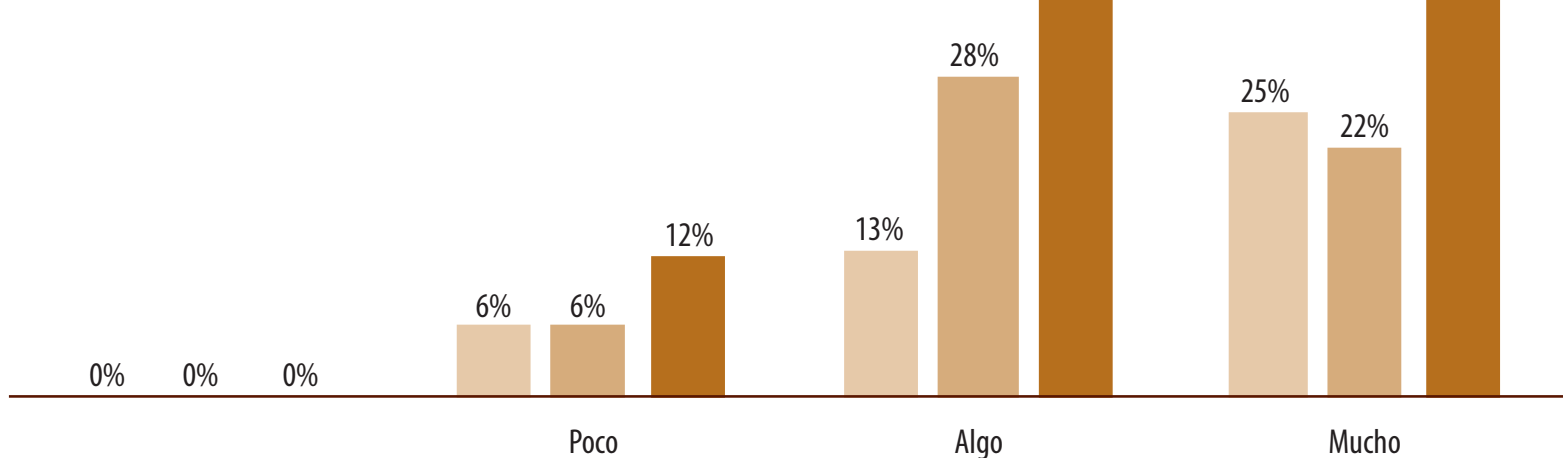

Figura 2. Sobre la experiencia del trabajo. Consulta realizada: ¿Considera que la experiencia al realizar el trabajo conjunto entre los dos cursos aportó a su formación como profesional en Ingeniería Química? 
doi: http://dx.doi.org/10.15359/ree.20-1.19

URL: http://www.una.ac.cr/educare

CORREO: educare@una.cr

La segunda pregunta realizada explora la opinión del estudiantado en cuanto a las habilidades generadas en su persona, esto en relación con su futura profesión (los resultados se muestran en la figura 3), a lo cual el $94 \%$ contestó afirmativamente y solo un $6 \%$ considera que la realización del trabajo no contribuyó a la adquisición de habilidades para su futura profesión. Esta pregunta, como todas las otras, se basa en la percepción estudiantil, lo cual es importante en tanto que la percepción estudiantil, en caso de ser positiva, funge como elemento motivador que da significado a las actividades realizadas, lo cual mantiene el interés del estudiantado. Se encuentra concordancia con las observaciones del equipo docente, pues como ya se mencionó, se percibió que el interés, con respecto a grupos de estudiantes que realizaban su proyecto final del curso en un mismo nivel, decae con el tiempo, mientras tanto, en este caso la motivación es sostenida gracias a la interacción de los niveles.

La última pregunta evalúa la percepción estudiantil en cuanto a si considera que es más provechoso realizar el trabajo en conjunto o de forma individual, a ello el $72 \%$ contestó que es más provechoso realizar el trabajo de forma conjunta, en tanto que un $19 \%$ considera que es igual y solo el $9 \%$ considera que es menos provechoso realizar el trabajo de una forma conjunta, estos resultados se muestran en la figura 4. Esta pregunta pretendía evaluar si el estudiantado consideraba que estaba obteniendo algún valor agregado a cambio del esfuerzo extra que conlleva el realizar el trabajo de forma conjunta. Dicho valor agregado se materializó, para los equipos de Análisis Gráfico, en una mejor comprensión del proyecto y una contextualización del aprendizaje, gracias a la cual lograron apropiarse de conceptos y conocimientos de un nivel avanzado de la carrera, esto fue corroborado por el equipo docente en las presentaciones de los avances, donde el lenguaje utilizado, así como las reacciones a las preguntas formuladas y la estructuración de las respuestas lo demostraron.

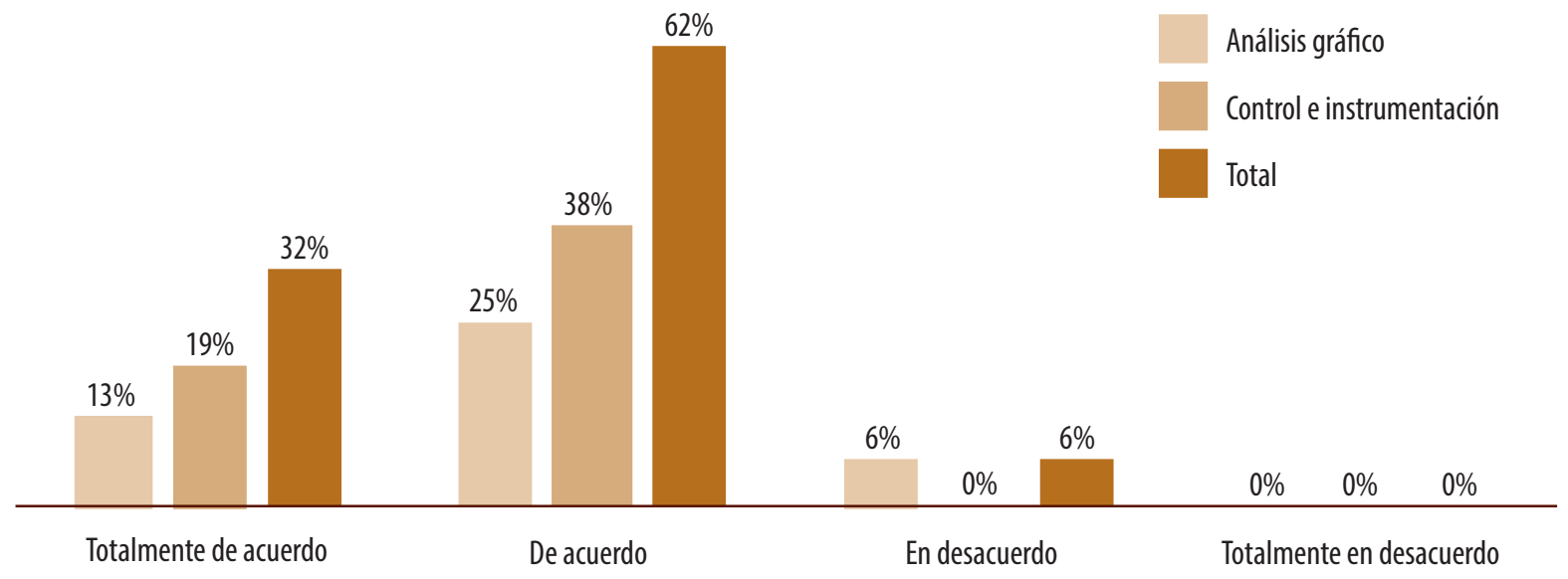

Figura 3. Sobre las habilidades adquiridas. Consulta realizada: ¿Cree que este trabajo le ha ayudado a desarrollar habilidades para su futura profesión? 
doi: http://dx.doi.org/10.15359/ree.20-1.19

URL: http://www.una.ac.cr/educare

CORREO: educare@una.cr

En cuanto a la pertinencia y tino de la estructura del trabajo y su extrapolación a otras áreas de la carrera, se realizaron dos consultas, la primera de ellas se muestra en la figura 5, en esta se consultó a la muestra de estudiantes sobre si consideran pertinente aplicar la modalidad del trabajo a otros cursos, a lo cual el 78 \% contestó afirmativamente. Esto implica que la percepción sobre la modalidad del trabajo es, en general, buena y que un alto porcentaje de estudiantes estaría dispuesto a experimentar experiencias similares en otros cursos de la carrera. Cabe señalar que al realizar el proyecto de forma conjunta, se incrementa el trabajo por parte de los equipos, por lo cual, el considerar que la estrategia puede ser aplicada a otros cursos de la carrera, a pesar de ese incremento de trabajo, muestra que los beneficios de la estrategia de interacción son visualizados por el estudiantado. Así se percibió por el equipo docente en las diversas observaciones realizadas, las cuales ya han sido discutidas.

Finalmente, se consultó sobre si realizaría el trabajo en conjunto o de forma individual, en caso de que se le diera a elegir, a lo cual el 72 \% contestó que de forma conjunta, en tanto que el $28 \%$ prefiere la modalidad tradicional (figura 6). En este caso, la consulta, al realizarse posterior a la finalización del trabajo, es un indicador de preferencia, o sea, el grupo de estudiantes prefieren, en su mayoría, la modalidad transgrupal para el desarrollo del trabajo por sobre la modalidad tradicional.

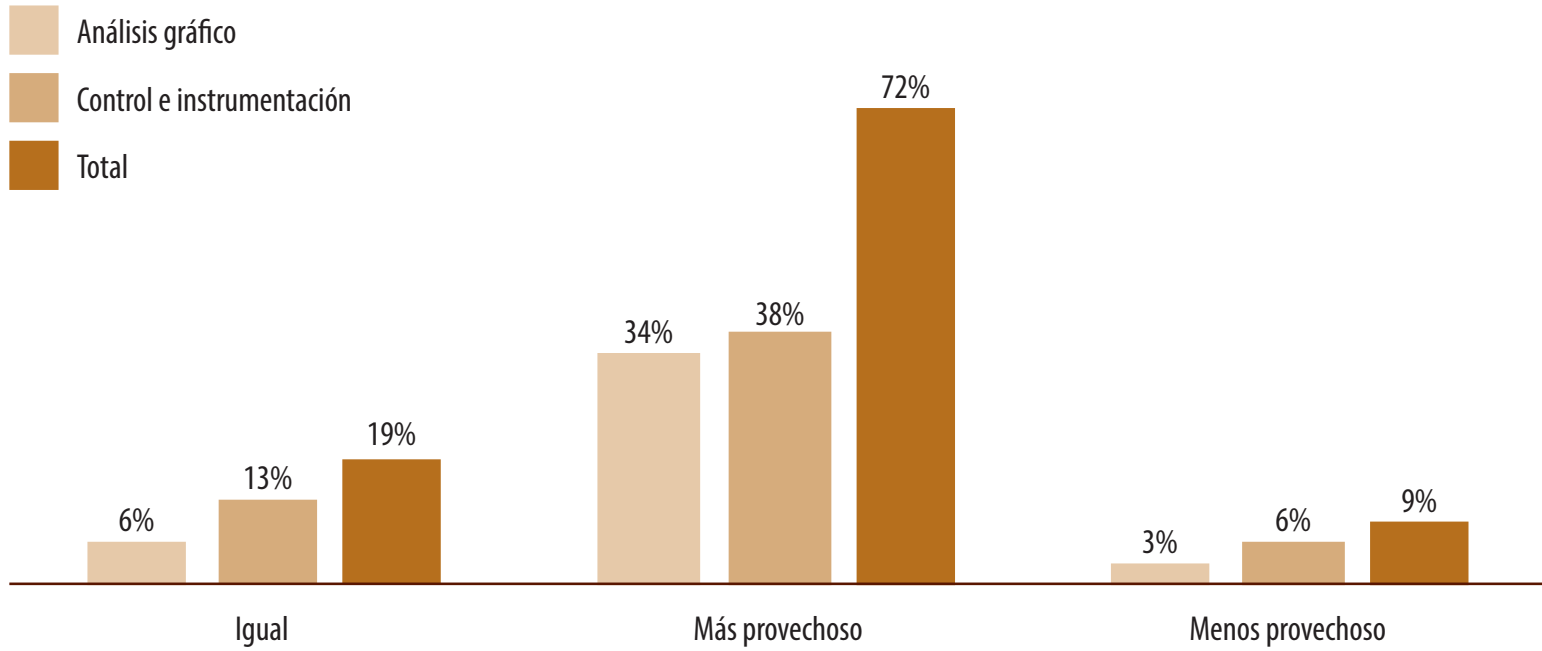

Figura 4. Respuesta a la consulta: ¿Siente que hacer el trabajo en conjunto es más o menos provechoso para su formación como profesional en Ingeniería Química que hacerlo solo con sus compañeros y compañeras de curso? 
doi: http://dx.doi.org/10.15359/ree.20-1.19

URL: http://www.una.ac.cr/educare

CORREO: educare@una.cr

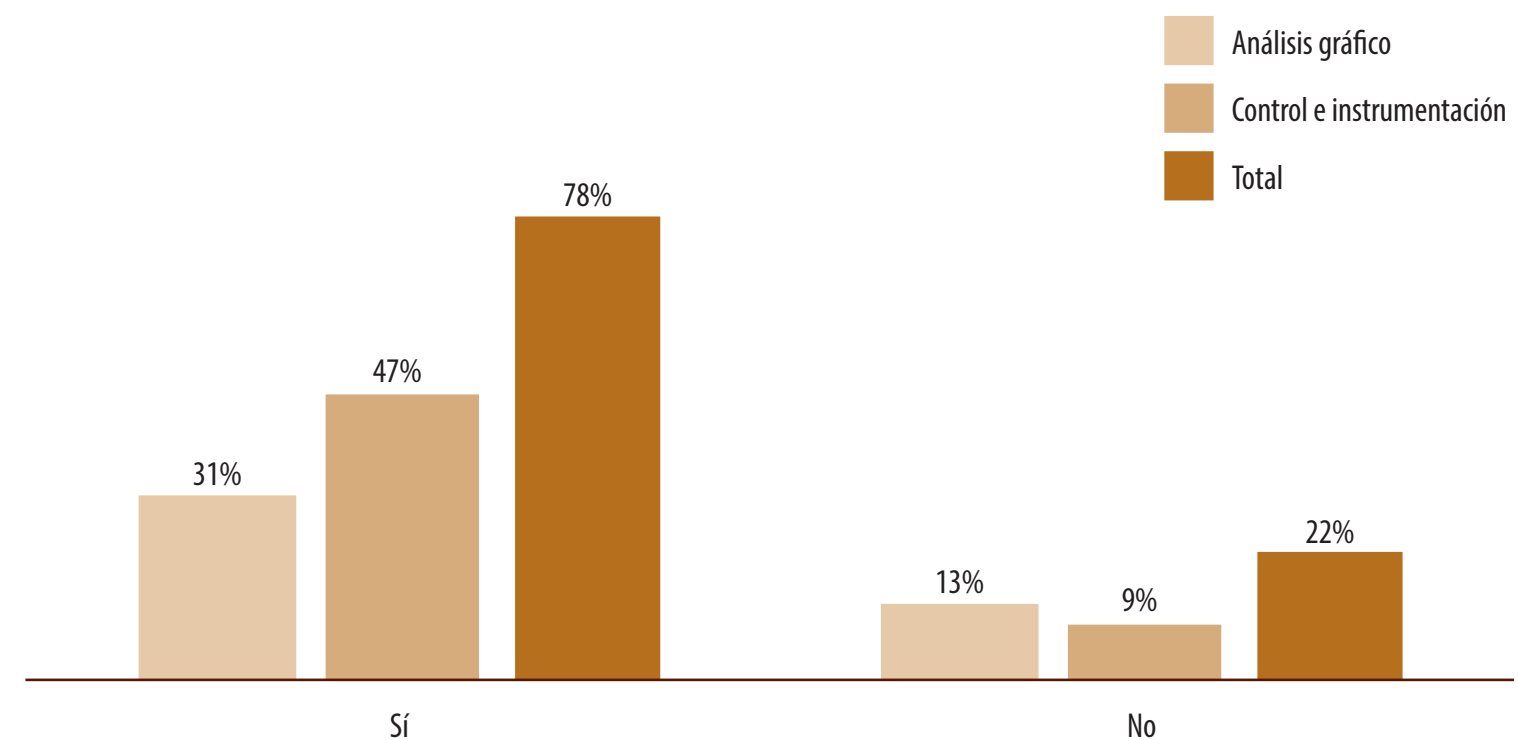

Figura 5. Opinión para extender la experiencia a otros cursos. Consulta realizada: ¿Considera adecuado aplicar este tipo de experiencia en otros cursos?

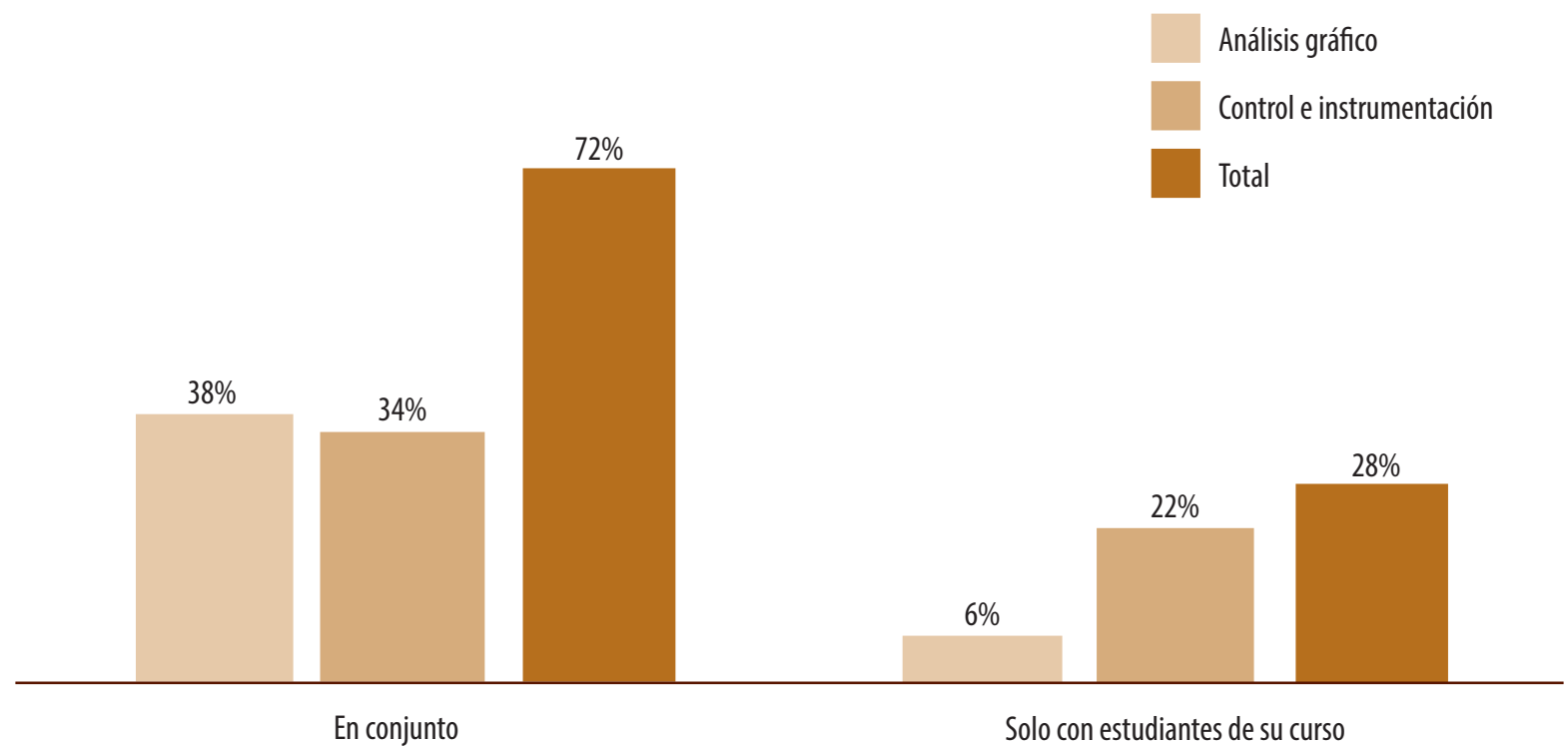

Figura 6. Respuesta a la consulta: ¿Si pudiera elegir, haría el trabajo en conjunto o solo con sus compañeros y compañeras de curso? 
doi: http://dx.doi.org/10.15359/ree.20-1.19

URL: http://www.una.ac.cr/educare

CORREO: educare@una.cr

\section{Impacto}

Para evaluar el impacto en cada participante en el trabajo, se realizó una pregunta abierta en la que se consultaba sobre lo que más le había gustado del trabajo, las respuestas fueron poco variadas, las opiniones se pueden resumir en un solo enunciado que, básicamente, engloba la opinión de la mayoría. En el caso del curso Análisis Gráfico, al 86 \% del estudiantado lo que más les gustó fue el Acercamiento a la carrera, compartir con personas de diferente nivel y la visión de futuro que les brindó la interacción.

En lo que respecta al estudiantado de Control e Instrumentación, las respuestas presentan una mayor diversidad; sin embargo, el $67 \%$ afirmó que lo mejor del trabajo había sido Colaborar con una generación nueva, el trabajo en equipo y poder explicar cosas. El restante $23 \%$ se divide en utilizar un simulador de procesos y resolver un problema de la vida real.

Así se observa que ambos grupos de estudiantes se ven beneficiados con el trabajo. Revela, además, que a la población estudiantil le agradó utilizar sus conocimientos, compartirlos, sentir que alguien más se ve beneficiado con ellos. La interacción transgrupal deja así un efecto sinérgico en el que el resultado de la experiencia es más que la simple suma de los conocimientos de cada grupo, es la generación de nuevas experiencias y capacidades más allá de los recursos de cada grupo por separado.

En una última etapa, se realizó una entrevista a dos estudiantes que participaron del estudio cuando se encontraban en Análisis Gráfico y en este momento se encuentran en su cuarto año de carrera. El objetivo de la entrevista se basó en cotejar la pregunta inicial del estudio (¿cómo influyó la interacción multinivel entre estudiantes de cursos de diferentes niveles?), además, de valorar el impacto luego de dos años de transcurrida la experiencia. A continuación se realiza un resumen de lo dicho por el estudiante y la estudiante entrevistada.

Al iniciar la entrevista se les planteó ¿qué les pareció la experiencia de interacción entre los cursos de Análisis Gráfico y Control e Instrumentación? Para lo que la estudiante expone que "La educación es un proceso, en cada etapa de la carrera universitaria se debe tener claro un panorama, no es ver toda la materia de una vez, pero sí tener un panorama para saber hacia dónde se dirige la formación". Esta idea, expresada por la estudiante, es acorde con lo expresado por Cross (1999): aprender se basa en hacer conexiones, al aislar los contenidos, como la estudiante lo percibe, no visualiza cómo dichos contenidos le serán de utilidad. Por su parte, el estudiante recalca que "la Ingeniería Química es abstracta, el concepto es complejo, el panorama aclararía al estudiantado hacia dónde van y por qué estudiar los temas que se ven en el momento". Ambas percepciones estudiantiles acerca de la problemática actual de la carrera se pueden resumir en lo expuesto por Illingworth (2010) sobre el gran aislamiento, dicho aislamiento temático genera esa "falta de panorama", como lo llaman en la entrevista. 
Respecto al proyecto realizado, la estudiante dice: “En el proyecto lo que más me gustó fue tener contacto con gente que me dijera que iba a hacer otras cosas, qué iba a ver en la carrera, yo pensaba que todo el mundo en Ingeniería Química trabajaba en calidad, a mí me gustaba pero veía que aquí solo se hacía eso y no había variedad. Investigué y en otros países se hacían otras cosas, pero hablando con los de Control e Instrumentación me dijeron en lo que realmente se trabajaba". Este evento no fue solo informativo para la estudiante, pues posteriormente afirma que "saber hacia dónde se dirige la carrera es un empujón para terminar con el ciclo básico de esta misma, porque una pasa por una crisis al llevar solo lo básico, pues no se entiende para qué le va a servir". De nuevo se reafirman los efectos del gran aislamiento de Illingworth (2010), que no solo limitan el potencial de aprendizaje (Efland, 2002), sino que influyen incluso en la motivación.

Pero los enunciados realizados por la parte estudiantil no solo evidencian la problemática, al introducir el concepto de "panorama", mencionado tanto por el estudiante como por la estudiante, se está planteando la solución, el "panorama" puede entenderse como la integración propuesta por Efland (2002) o las conexiones mencionadas por Cross (1999).

Con base en esta visión de "panorama", ambos estudiantes comentaron sobre proyectos que actualmente tienen para "integrar estudiantes de segundo y tercer año de la carrera con estudiantes de cuarto y quinto" en un proyecto que se está gestando en la Facultad de Ingeniería de la Universidad de Costa Rica. "Nos parece muy adecuado que haya una mezcla de información" comenta el estudiante. Ante estas afirmaciones, la profesora consulta sobre la opinión que hoy, dos años después de haber realizado el proyecto conjunto, tienen. "Eso fue el inicio de todo esto que estamos pensando", comenta la estudiante, "Fue la primera vez que me enfrenté a una experiencia en la que las cosas se salían de nuestras manos, el grupo era problemático, no eran nuestros amigos de siempre, tuvimos problemas, pero nos reunimos, hablamos y establecimos reglas", comenta por su parte el estudiante. Estas afirmaciones remiten, desde nuestro punto de vista, a lo expuesto por Pardini (2005) sobre las interacciones multinivel, sobre lo cual Pardini (2005) expone que generan mejores habilidades afectivas, sociales y de liderazgo que los sistemas tradicionales.

Al finalizar la entrevista percibimos que la experiencia del trabajo interactivo resultó altamente significativa para ambos estudiantes, no solo desde la comprensión de los contenidos en el momento de llevar el curso, sino porque, a partir de dicha experiencia, ambos estudiantes están gestionando proyectos en los cuales poner en contacto a las personas en la Escuela de Ingeniería Química, ya no solo a estudiantes de diferentes niveles, sino también al cuerpo docente. A partir de la experiencia interactiva se generó una apertura (como se expresa en la entrevista), gracias a la cual están estructurando ideas y proponiendo proyectos para extender la experiencia. Ambos estudiantes identificaron los problemas presentes en la carrera, debido a su estructura curricular, y encontraron en la interacción la respuesta a dichos problemas. 
doi: http://dx.doi.org/10.15359/ree.20-1.19

URL: http://www.una.ac.cr/educare

CORREO: educare@una.cr

Cabe mencionar que tanto el estudiante como la estudiante están gestionando los proyectos mencionados desde sus puestos en la Asociación de Estudiantes de Ingeniería Química, donde han adoptado un papel de líderes estudiantiles "estábamos hartos de criticar así que decidimos hacer algo", nos comentaron.

\section{Conclusiones}

La principal preocupación del equipo docente en esta investigación radicaba en la formación de islas de conocimiento, en el aislamiento generado por no existir interacción entre los niveles de una misma carrera universitaria. Se encontró que muchos autores se han referido al tema y lo han estudiado en niveles de primaria y actualmente en la universidad (Pardini, (2005); Pop-lliev y Nokleby (2011); Hickman y Kiss (2010); Kapadia (2010); Gardner et al. (2010). Se evidenció que la interacción puede potenciar el aprendizaje y, además, influir y contribuir en la generación y desarrollo de habilidades no formales, como son: el trabajo en equipo, el liderazgo, la motivación hacia la carrera profesional, fortalecimiento de los vínculos afectivos con las personas, las habilidades sociales, el diálogo, el consenso, etc.

Con el fin de evaluar la pregunta que generó el estudio, ¿cómo influye la interacción multinivel, entre estudiantes de cursos de diferentes niveles de la carrera de Ingeniería Química, en el aprendizaje y las habilidades desarrolladas por los grupos de estudiantes?, se realizó una estrategia didáctica que consistió en la unión de dos cursos de niveles distintos para realizar un proyecto final en conjunto, se escogió un curso en segundo año y otro de quinto para el estudio. Se utilizaron varios instrumentos, principalmente la observación y las reuniones en avances, además del recabo de información de primera mano por medio de una bitácora que llevaba cada equipo de trabajo a manera de diario.

A lo largo de este artículo se discutió acerca de la conformación de los conocimientos de ambos cursos, lo cual planteaba el primer problema a resolver, pues en segundo año el estudiantado se inicia en los conceptos principales de su futura profesión, mientras en quinto la dinámica permite problemas de mayor complejidad y abstracción. Por tanto, se debió afrontar la necesidad de realizar medios para comunicar los conceptos que para unos fueron complejos y para otros ya obvios; se encontró que los grupos resolvieron este primer conflicto generando su propio lenguaje y buscando espacios para reuniones. Es importante recalcar que se notó una generación de lenguaje técnico y una apropiación de los conceptos abstractos entre el estudiantado de segundo año, lo cual evidenció que la interacción permitió un avance significativo en ese aspecto, que sorprendió al equipo docente, pues, por lo general, no ocurre hasta llegados a cuarto y quinto año.

Por otro lado, se presentaron problemas de índole personal y de equipo, como la planificación y el consenso en las reuniones, que los grupos pudieron resolver por medio de 
sus propios instrumentos como lo fueron grupos en Facebook, chat de videos y reuniones semanales presenciales. Para el equipo docente esto evidenció el desarrollo de habilidades de trabajo en equipo y de habilidades de comunicación que de otra manera no se daban entre estos dos niveles.

Así mismo, el estudiantado de quinto año expresó que habían adquirido conciencia de lo que habían avanzado en la carrera, aprendió a resolver y sobrepasar el nivel del lenguaje técnico para expresarlo de manera sencilla y se les colocó en una situación similar a la vida profesional.

Ambos niveles se encontraron, en su mayoría, mucho más motivados a lo largo del proyecto, lo que finalizó en trabajos de alta calidad en cuanto a lo académico y en vínculos afectivos con personas de distintos niveles, formando amistades y espacios de conversación que sobrepasaron lo formal para integrar otras habilidades sociales.

En el cuestionario realizado a 32 estudiantes se encontró, primordialmente, que a pesar de las labores extra, la gran mayoría de estudiantes considera que el trabajo realizado es más provechoso al realizarse en conjunto que si se hiciera con equipos de trabajo compuestos por estudiantes del mismo nivel, además, la mayoría prefiere que se realice el trabajo en conjunto y considera que esta metodología es adecuada para su formación.

En cuanto a la entrevista a dos estudiantes, dos años después de haber realizado la experiencia, se encontró que la estrategia de interacción impulsó acciones similares de interacción multinivel que, desde su posiciones de líderes estudiantiles, pretenden ejecutar y se encuentran gestionando para la resolución de los problemas que como estudiantes han identificado en su proceso de formación, reconociendo que el proyecto de Análisis y Control fue su principal incentivo.

Por último, para el equipo docente, la interacción transgrupal dejó un efecto sinérgico en el que el resultado de la experiencia es más que la simple suma de los conocimientos de cada grupo, es la generación de nuevas experiencias y capacidades.

La principal recomendación del estudio se genera a partir de los hallazgos, donde se encontró que una manera de abordar las llamadas islas de conocimientos es atacarlas a partir de la generación de espacios donde el estudiantado de la carrera se encuentre en contacto con sus homólogos de distintos niveles, se pueda compartir experiencias y trascender los niveles de conocimientos que genera la malla curricular como se encuentra planteada. Por lo que es importante generar estos espacios, no solo entre niveles de estudiantes, sino también con el cuerpo docente y profesionales en el área en que se están preparando, para potenciar los conocimientos y no limitar la velocidad con la que una persona pueda interesarse por conceptos a niveles superiores. 
doi: http://dx.doi.org/10.15359/ree.20-1.19

URL: http://www.una.ac.cr/educare

CORREO: educare@una.cr

\section{Referencias}

Anderson, R. H. (Febrero, 1987). Shaping up the shop: how school organization influences teaching and learning [Ordenando la casa: Cómo la organización de las escuelas influencia la enseñanza y el aprendizaje]. Educational Leadership, 44(5), 44-46.

Carter, P. (Setiembre, 2005). The modern multi-age classroom [La clase multiedad moderna]. El Educational Leadership, 63(1), 54-58. Recuperado de http://www.ascd.org/publications/ educational leadership/sept05/vol63/num01/The Modern Multi-Age Classroom.aspx

Cross, K. P. (Junio, 1999). Learning is about making. [El aprendizaje se trata de hacer conecciones]. The Cross Papers, 3, 5-28. Recuperado de http://djames84.net/Cert 51/Learning Is About Connections.pdf

Efland, A. D. (2002). Art and cognition: Integrating the visual arts in the curriculum [Arte y cognición: La integración de las artes visuales en el currículo]. New York: Teachers College Press.

Gardner, D. S., Tuchman, E.y Hawkins, R. (2010). Teaching note: A cross-curricular, problem-based project to promote understanding of poverty in urban communities [Proyecto basado en problemas con curricula transversal para mejorar el entendimiento de la pobreza en comunidades urbanas]. Journal of Social WorkEducation, 46(1), 147-156. doi: http://dx.doi. org/10.5175/JSWE.2010.200800094

Hickman, R. y Kiss, L. (2010). Cross-curricular gallery learning: A phenomenological case study [Aprendizaje en galería con curricula transversal: Estudio fenomenológico de caso]. The International Journal of Art \& Design Education, 29(1), 27-36. doi: http://doi.org/10.1111/ j.1476-8070.2010.01635.x

Illingworth, S. (Marzo, 2010). From "splendid isolation" to productive alliances: Developing meaningful cross-curricular approaches [Del "gran aislamiento" hacia alianzas productivas: Desarrollo de aproximaciones significativas con curricula transversal]. Teaching History, $138,42-46$.

Kapadia, R. (2010). Promoting a cross-curricular pedagogy of risk [Promoción de una pedagogia del riesgo de curricula transversal]. Mathematics Teaching, 219, 44-27.

Lauer, P. A. (2000). Instructional practices and implementation issues in multiage classrooms [Las prácticas de enseñanza y los detalles de su implementación en clases multiedad]. Recuperado de http://files.eric.ed.gov/fulltext/ED450099.pdf

Pardini, P. (Marzo, 2005). The slowdown of the multiage classroom [La desaceleración de la clase multiedad]. School Administrator, 62(3), 22-30. 
Pop-lliev, R. y Nokleby, S. B. (2011). Cross-course integrated group design projects: $1+1=11$ [Proyectos de diseño en grupos integrados con transversalidad de cursos: $1+1=11$ ]. Proceedings of the Canadian Engineering Education Association, 303-308. Recuperado de http://library.queensu.ca/ojs/index.php/PCEEA/article/view/3841

Posner, G. J. (1974). The extensiveness of curriculum structure: A conceptual scheme [La amplitud de la estructura curricular: Un esquema conceptual]. Review of Educational Research, 44(4), 401-407. doi: http://dx.doi.org/10.3102/00346543044004401

\section{Cómo citar este artículo en APA:}

Calderón-Castro, J. A. y Garro-Mena, L. (Enero-abril, 2016). Interacción multinivel en estudiantes de Ingeniería Química de la Universidad de Costa Rica. Revista Electrónica Educare, 20(1), 1-21. doi: http://dx.doi. org/10.15359/ree.20-1.19

Nota: Para citar este artículo en otros sistemas puede consultar el hipervínculo "Como citar el artículo" en la barra derecha de nuestro sitio web: http://www.revistas.una.ac.cr/index.php/EDUCARE/index 